\title{
PHASE SHIFT OF ISOSPECTRAL POTENTIALS
}

\author{
Sabyasachi Mahapatra \\ Department of Physics, St. Paul's Cathedral Mission College \\ 33/1, Raja Rammohan Roy Sarani, Kolkata-700 009, India \\ E-mail: smahapatra99@gmail.com \\ (Received by April 10, 2017; in final form - May 22, 2017)
}

\begin{abstract}
The phase shift produced by isospectral potentials has been critically investigated. The isospectral potentials may look quite different in shape from the original potentials although they have identical spectra. In this work, it is shown that the isospectral potentials produce same phase shift with the original potentials at a given energy.
\end{abstract}

Key words: phase shift, supersymmetry, isospectral potential.

PACS number(s): 03.65.-w, 03.65.Nk

\section{INTRODUCTION}

During the past thirty years, the ideas of Supersymmetry for understanding potential problems in Quantum Mechanics have been developed using some ideas borrowed from Quantum Field theory. Supersymmetric Quantum Mechanics (SSQM) was introduced by Witten [1], Coopper and Freedman [2] as a simple model for testing supersymmetric field theories which were introduced by Gol'fand and Likhtman [3] for a unified description of the fundamental interactions in nature. The study of SSQM provided an insight into the factorization of the Hamiltonian and introduction of partner Hamiltonians having wholly or partially identical energy spectrum. It also explained analytical solvability of a class of potentials, whose partner potentials have the same shape, thus introducing the concept of shape invarince.

From a given Hamiltonian $\left(H^{(1)}\right)$, a partner Hamiltonian $\left(H^{(2)}\right)$ is usually obtained by deleting the ground state of $H^{(1)}$, the rest of the spectrum of $H^{(1)}$ being identical with that of $H^{(2)}$ [4]. The partner Hamiltonian $H^{(2)}$ can also be obtained by adding an energy level below the ground state of $H^{(1)}$ or by making the spectrum of $H^{(2)}$ identical with that of $H^{(1)}$ (isospectral Hamiltonians). The principal result is the energy degeneracy, viz., $E_{n+1}^{(1)}=E_{n}^{(2)},(n=0,1,2, \ldots)$, where $E_{n}^{(i)}$ is the energy of the $n$-th excited (bound) state of $H^{(i)}(i=1,2)$.

Now, if $V^{(2)}$ of the partner Hamiltonian $H^{(2)}$ has the same functional shape (but with different parameters $a_{2}$ and $a_{1}$ ) as that of the potential $V^{(1)}$ of the original Hamiltonian $H^{(1)}$, i.e.

$$
\begin{array}{r}
V^{(2)}\left(r ; a_{1}\right)=V^{(1)}\left(r ; a_{2}\right)+R\left(a_{1}\right), \\
\text { where } a_{2}=f\left(a_{1}\right)
\end{array}
$$

(the remainder $R\left(a_{1}\right)$ being independent of $r$ ), then the potential is said to be a 'shape invariant potential (SIP)'. Then the entire bound state energy spectrum and corresponding energy eigen functions of $H^{(1)}$ can be obtained algebraically [4]. It is generally believed that the results of all observables can be obtained algebraically for SIPs.

The above procedure can also be extended to the scattering (unbound) states. From the asymptotic form of the scattering wave functions, one can show that the phase shift $\delta_{l}^{(1)}(k)$ of the $l$-th partial wave in $V^{(1)}$ bears a simple relation with the phase shift $\delta_{l+1}^{(2)}(k)$ of the $(l+1)$-th partial wave in $V^{(2)}$ [5]

$$
\delta_{l+1}^{(2)}(k)=\delta_{l}^{(1)}(k)-\tan ^{-1}\left(\frac{\gamma_{0}}{k}\right)
$$

where $k$ is the wave number of the incident wave and $\gamma_{0}=\sqrt{\frac{2 m B_{0}}{\hbar^{2}}}, B_{0}$ being the binding energy of the ground state in $V^{(1)}$. An exhaustive study $[6,7]$ has been done to examine the validity of Eq. (2) for Supersymmetric partner potentials. It has been established that Eq. (2) holds for both SIP and non-SIP, which relates the phase shift produced by the $l$-th partial wave of $V^{(1)}(r)$, i.e. by the potential $V_{\mathrm{eff}}^{(1)}(r)=V^{(1)}(r)+\frac{\hbar^{2}}{2 m} \frac{l(l+1)}{r^{2}}$ and that by its SUSY partner $V_{\text {eff }}^{(2)}(r)$. The latter phase shift corresponds to the $(l+1)$-th partial wave. However, there is no relation between phase shifts produced by $V_{\text {eff }}^{(1)}(r)$ and $V^{(2)}(r)+\frac{\hbar^{2}}{2 m} \frac{(l+1)(l+2)}{r^{2}}$, where $V^{(2)}(r)$ is the SUSY partner of $V^{(1)}(r)$. It would be wrong to take $\delta_{l+1}^{(2)}(k)$ to be the phase shift produced by the $(l+1)$-th partial wave in $V^{(2)}(r)$. Numerical calculations of the phase shifts produced by a non-SIP as well as an SIP confirm this [7]. Since the phase shift relation is true for any pair of partner potentials, the phase shift relation [Eq. (2)] is well obeyed by all shape invariant, as well as non-shape invariant potentials. It is also shown that the phase shifts of shape invariant potentials can be derived analytically only if the potential together with the centrifugal repulsion corresponding to the orbital angular momentum $l$ is shape invariant and its partner involves centrifugal repulsion corresponding to orbital angular momentum $(l+1)$. Among the known SIPs related by translation of parameters [4], which have continuum spectra, only the Coulomb potential satisfies this criterion [6]. Except the Coulomb potential, no other SIP has the orbital angular momentum $l$ as the shape invariance parameter. Hence, even though $V^{(1)}$ is shape invariant and both equations (1) and (2) hold, the phase shift cannot be obtained algebraically for these potentials. But the phase shift relation 
is neither verified nor any research on scattering properties of isospectral potentials has been reported so far. If two different potentials have completely identical spectra, but differ in their shapes, they are called isospectral potentials [4]. As the isospectral potentials have identical eigenvalue, reflection and transmission coefficient, it will be very much interesting to know the phase shift relation between them. In this work, the phase shift produced by isospectral potentials has been examined critically for a finite square well potential.

The paper is organised as follows. In Sec. II, a theoretical framework for obtaining the isospectral potential along with phase shift is presented. In Sec. III, the results of the calculation are shown and discussed. Finally in Sec. IV, the conclusion is drawn.

\section{ISOSPECTRAL POTENTIAL AND CALCULATION OF PHASE SHIFT}

\section{A. Isospectral potential}

Using the idea of SSQM one can construct a partner potential for any given potential, so that the spectrum of the partner potential is identical to the original, except that the ground state of the original is missing in the spectrum of the partner potential. Using this procedure one can construct a family of strictly isospectral potentials $\hat{V}(r, \lambda)$, depending on a parameter $\lambda$ so that the potential has eigenvalue, reflection and transmission coefficient identical to those for the original potential $V(r)$. The potentials $V(r)$ and $\hat{V}(r, \lambda)$ may look quite different but are strictly isospectral. In the following, it will be discussed briefly regarding the construction of an isospectral potential [4] from any given potential $V_{\text {eff }}(r)$, which has a normalized ground state $\psi_{0}(r)$ with energy $E_{0}$. The potential $V_{\text {eff }}(r)$ may contain the centrifugal repulsion term corresponding to the orbital angular momentum $l$, which is assumed to be a good quantum number. Then $\psi_{0}(r)$ is the ground state of the subset of states with the orbital angular momentum $l$. The Schrödinger equation satisfied by $\psi_{0}(r)$ is

$$
\left[-\frac{\hbar^{2}}{2 m} \frac{d^{2}}{d r^{2}}+V_{\mathrm{eff}}(r)\right] \psi_{0}(r)=E_{0} \psi_{0}(r) .
$$

A superpotential $W(r)$ for this subset of states is defined as

$$
W(r)=-\frac{\hbar}{\sqrt{2 m}} \frac{\psi_{0}^{\prime}(r)}{\psi_{0}(r)} .
$$

Then $V_{\text {eff }}(r)$ can be expressed by

$$
V_{\mathrm{eff}}(r)-E_{0}=W^{2}(r)-\frac{\hbar}{\sqrt{2 m}} W^{\prime}(r),
$$

and one can define a supersymmetric partner potential $V_{\mathrm{eff}}^{(2)}(r)$ through

$$
V_{\mathrm{eff}}^{(2)}(r)-E_{0}=W^{2}(r)+\frac{\hbar}{\sqrt{2 m}} W^{\prime}(r),
$$

such that $V_{\text {eff }}(r)$ and its partner $V_{\text {eff }}^{(2)}(r)$ have identical energy spectra, except that the ground state of $V_{\text {eff }}(r)$ is missing in the spectrum of its partner [4]. Note that Eq. (6) may be considered as a non-linear differential equation for $W(r)$ for a given $V_{\mathrm{eff}}^{(2)}(r)$. Now, Eq. (6) being non-linear, its solution is not unique. The most general super potential $\hat{W}$ can be obtained $[4,8]$, such that

$$
V_{\mathrm{eff}}^{(2)}(r)-E_{0}=\hat{W}^{2}(r)+\frac{\hbar}{\sqrt{2 m}} \hat{W}^{\prime}(r) .
$$

Now, for the given partner potential $V_{\mathrm{eff}}^{(2)}(r)$, Eq. (7) (called Riccati equation) can be considered as a nonlinear differential equation for the function $\hat{W}(r)$. One can reconstruct $V_{\text {eff }}(r)$ with this solution, using Eq. (5). But solution of the non-linear Eq. (7) is not unique, its most general solution being [4] (for simplicity in a theoretical calculation, we choose the units in the way that $\frac{\hbar}{\sqrt{2 m}}=1$. Then the factor $\frac{\hbar}{\sqrt{2 m}}$ in Eqs. (3)-(7) is replaced by 1 )

$$
\hat{W}(r ; \lambda)=W(r)+\frac{d}{d r} \ln \left|I_{0}(r)+\lambda\right|,
$$

where $\lambda$ is an integration constant and the function $\hat{W}(r ; \lambda)$ depends parametrically on $\lambda ; I_{0}(r)$ is given by

$$
I_{0}(r)=\int_{0}^{r}\left[\psi_{0}\left(r^{\prime}\right)\right]^{2} d r^{\prime}
$$

Then the family of potentials

$$
\begin{aligned}
\hat{V}_{\mathrm{eff}}(r ; \lambda) & =\hat{W}^{2}(r ; \lambda)-\hat{W}^{\prime}(r ; \lambda)+E_{0} \\
& =V_{\mathrm{eff}}(r)-2 \frac{d^{2}}{d r^{2}} \ln \left|I_{0}(r)+\lambda\right|
\end{aligned}
$$

(with permissible values of $\lambda$ ), has the same partner $V_{\text {eff }}^{(2)}(r)$. Therefore, the potentials $\hat{V}_{\text {eff }}(r ; \lambda)$ given by Eq. (10) with any permissible values of $\lambda$, have the spectrum identical to that of $V_{\text {eff }}(r)$. Since $\psi_{0}(r)$ is normalized, $0 \leq I_{0}(r) \leq 1$. Hence from Eq. (8), one notices that the interval $-1 \leq \lambda \leq 0$ will lead to divergences and hence not allowed. For all other values of $\lambda, \hat{V}_{\text {eff }}(r ; \lambda)$ is strictly isospectral with $V_{\text {eff }}(r)$.

\section{B. Numerical calculation of the phase shift}

For the potential $V(r)$ [with $V_{\infty}\left(\lim _{r \rightarrow \infty} V(r)=V_{\infty}\right)$ subtracted, so that $V(r)$ vanishes asymptotically] the radial Schrödinger equation for the $l$-th partial wave takes the form

$$
\left[-\frac{\hbar^{2}}{2 m} \frac{d^{2}}{d r^{2}}+V_{\mathrm{eff}}(r)-E\right] \psi_{E}^{(1)}(r)=0,
$$

Where $V_{\text {eff }}(r)=V(r)+\frac{\hbar^{2}}{2 m} \frac{l(l+1)}{r^{2}}$. The asymptotic solution of Eq. (11) can be expressed as $\psi_{E}^{(1)}(r) \propto \sin [k r-$ $\left.\frac{l \pi}{2}+\delta_{l}^{(1)}(k)\right]$, where $k=\sqrt{\frac{2 m E}{\hbar^{2}}}$. Eq. (11) can be solved 
numerically using the Runga-Kutta algorithm from $r \rightarrow$ 0 (subject to $\psi_{E}^{(1)}(0)=0$ ) to two large values of $r$ (say $R_{1}$ and $\left.R_{2}\right)$ to obtain $\phi_{1}=\psi_{E}^{(1)}\left(R_{1}\right)$ and $\phi_{2}=\psi_{E}^{(1)}\left(R_{2}\right)$. Then

$$
\delta_{l}^{(1)}(k)=\tan ^{-1}\left[\frac{\phi_{2} \sin \left(k R_{1}-\frac{l \pi}{2}\right)-\phi_{1} \sin \left(k R_{2}-\frac{l \pi}{2}\right)}{\phi_{1} \cos \left(k R_{2}-\frac{l \pi}{2}\right)-\phi_{2} \cos \left(k R_{1}-\frac{l \pi}{2}\right)}\right] .
$$

For calculation of the phase shift for the isospectral potential, the above procedure can be followed, replacing $V_{\text {eff }}(r)$ by $\hat{V}_{\text {eff }}(r, \lambda)$ in Eq. (11).

\section{Application for finite square well potential}

The above-mentioned procedure can be applied for a three dimensional finite square well potential as a simple demonstrative application. The square well potential has a depth $V_{0}$ and range $R$ :

$$
\begin{aligned}
V(r) & =-V_{0}, & & r \leq R \\
& =0, & & r>R .
\end{aligned}
$$

The finite square well potential is not an SIP, although it is solvable semi-analytically. The ground and other state wave functions in this well can be expressed in terms of standard mathematical functions. Writing the bound state wave functions as

$$
\Psi_{n l m}(\mathbf{r})=R_{n l}(r) Y_{l m}(\theta, \phi)
$$

for potential (13):

$$
\begin{aligned}
R_{n l}(r) & =N_{n l} j_{l}(K r), & & r \leq R \\
& =N_{n l} \frac{j_{l}(K R)}{k_{l}(k R)} k_{l}(k r), & & r>R,
\end{aligned}
$$

where $j_{l}(x)$ is the spherical Bessel function and $k_{l}(x)$ is the modified spherical Bessel function that is regular in the asymptotic region [9]. $k$ and $K$ are given by

$$
k=\sqrt{-\frac{2 m E_{n l}}{\hbar^{2}}} \quad K=\sqrt{\frac{2 m}{\hbar^{2}}\left(V_{0}+E_{n l}\right)},
$$

with $E_{n l}<0$.

The normalization constant is given by

$$
N_{n l}=\left[\int_{0}^{R}\left[j_{l}(K r)\right]^{2} r^{2} d r+\left(\frac{j_{l}(K R)}{k_{l}(k R)}\right)^{2} \int_{R}^{\infty}\left[k_{l}(k r)\right]^{2} r^{2} d r\right]^{-\frac{1}{2}}
$$

The quantity $I_{0}(r)$ in Eq. (9) can be calculated as

$$
\begin{aligned}
I_{0}(r) & =\left(N_{n l}\right)^{2} \int_{0}^{r}\left[j_{l}\left(K r^{\prime}\right)\right]^{2} r^{\prime 2} d r^{\prime}, \quad(\text { for } r \leq R), \\
& =\left(N_{n l}\right)^{2}\left[\int_{0}^{R}\left[j_{l}\left(K r^{\prime}\right)\right]^{2} r^{\prime 2} d r^{\prime}+\left(\frac{j_{l}(K R)}{k_{l}(k R)}\right)^{2} \int_{R}^{r}\left[k_{l}\left(k r^{\prime}\right)\right]^{2} r^{\prime 2} d r^{\prime}\right] \quad(\text { for } r>R) .
\end{aligned}
$$

Substituting Eq. (18) into Eq. (10), the one parameter family of isospectral potentials can be obtained as

$$
\begin{aligned}
\hat{V}_{\text {eff }}(r ; \lambda) & =\frac{\hbar^{2}}{2 m}\left[-V_{0}+\frac{l(l+1)}{r^{2}}-\frac{4}{[I(r)+\lambda]}\left(N_{n l}\right)^{2} r j_{l}(K r)\left[j_{l}(K r)+K r j_{l}^{\prime}(K r)\right]\right. \\
& \left.+\frac{2}{[I(r)+\lambda]^{2}} r^{4}\left(N_{n l}\right)^{4}\left(j_{l}(K r)\right)^{4}\right], \quad(\text { for } \quad r \leq R), \\
\hat{V}_{\text {eff }}(r ; \lambda) & =\frac{\hbar^{2}}{2 m}\left[\frac{l(l+1)}{r^{2}}-\frac{4}{[I(r)+\lambda]}\left(N_{n l} \frac{j_{l}(K R)}{k_{l}(k R)}\right)^{2} r k_{l}(k r)\left[k_{l}(k r)+k r k_{l}^{\prime}(k r)\right]\right. \\
& \left.+\frac{2}{[I(r)+\lambda]^{2}} r^{4}\left(N_{n l}\right)^{4}\left(k_{l}(k r)\right)^{4}\left(\frac{j_{l}(K R)}{k_{l}(k R)}\right)^{4}\right] \quad(\text { for } r>R) .
\end{aligned}
$$

The normalization constant $N_{n l}$ is given by Eq. (17). For a chosen value of $l, E_{n l}$ corresponds to the ground state energy $(n=1)$ and $k$ and $K$ are given by Eq. (16) with this energy. 


\section{RESULTS AND DISCUSSION}

For numerical calculation, $V_{0}$ is chosen to be 200 $\mathrm{MeV}, a=2.0 \mathrm{fm}, \frac{\hbar^{2}}{2 m}=20.735 \mathrm{MeV} \mathrm{fm}^{2}$ and $l=1$. With these parameters, there is only one bound state (ground state). The energy equation (continuity of the log-derivative of the wave function) is solved numerically to obtain the ground state energy as $E_{11}=-123.803870$ $\mathrm{MeV}$. Next $I_{0}(r)$ is calculated using Eq. (18) and hence $\hat{V}_{\text {eff }}(r ; \lambda)$ is evaluated from Eq. (19). $\hat{V}_{\text {eff }}(r ; \lambda)$ against $r$ is plotted in Fig. 1 for various values of $\lambda$, viz., $\lambda=0.1,0.01,0.001,0.0001$.

\begin{tabular}{|l|l|l|l|l|l|}
\hline Energy $(E)$ & Phase shift for & \multicolumn{4}{|c|}{ Phase shift for $\hat{V}_{\text {eff }}(r ; \lambda)$} \\
\cline { 2 - 7 } (in MeV) & $V_{\text {eff }}(r)$ & $\lambda=0.1$ & $\lambda=0.01$ & $\lambda=0.001$ & $\lambda=0.0001$ \\
\hline 0.10 & -1.510250 & -1.509612 & -1.509535 & -1.509511 & -1.509511 \\
0.20 & -1.508256 & -1.506125 & -1.505866 & -1.505786 & -1.505786 \\
0.30 & -1.493313 & -1.488633 & -1.488055 & -1.487876 & -1.487876 \\
0.40 & -1.466938 & -1.458182 & -1.457091 & -1.456751 & -1.456751 \\
0.50 & -1.426455 & -1.411337 & -1.409428 & -1.408830 & -1.408830 \\
0.60 & -1.371780 & -1.346808 & -1.343611 & -1.342602 & -1.342602 \\
0.70 & -1.298654 & -1.258510 & -1.253290 & -1.251635 & -1.251635 \\
0.80 & -1.201339 & -1.138187 & -1.129850 & -1.127194 & -1.127194 \\
0.90 & -1.072862 & -0.976392 & -0.963550 & -0.959442 & -0.959442 \\
1.00 & -0.906852 & -0.767294 & -0.748860 & -0.742956 & -0.742956 \\
\hline
\end{tabular}

Table 1. Phase shift produced by the original and isospectral potential at different energies for different values of $\lambda$.

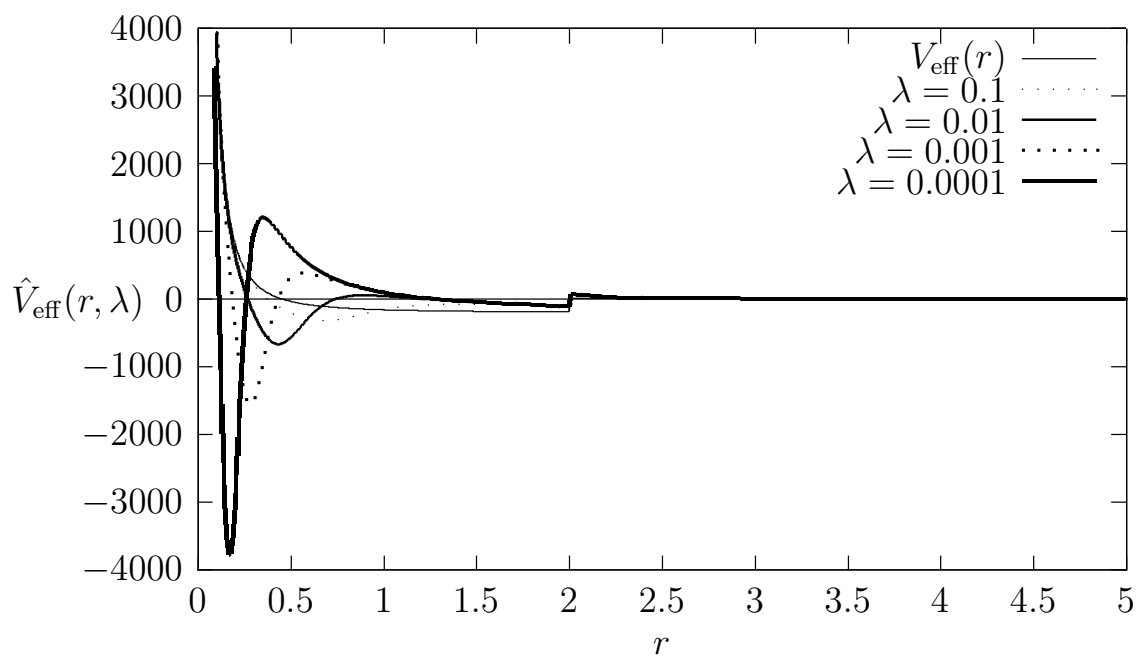

Fig. 1. Plot of $\hat{V}_{\mathrm{eff}}(r ; \lambda)$ (in $\mathrm{MeV}$ ) versus $r$ (in fm) for different values of $\lambda$ along with original potential $V_{\mathrm{eff}}(r)$.

The original potential $V_{\text {eff }}(r)$ is also plotted in the same figure and it is observed that $\hat{V}_{\text {eff }}(r ; \lambda)$ and $V_{\text {eff }}(r)$ differ in their shapes. $\hat{V}_{\text {eff }}(r ; \lambda)$ has a very deep and narrow well near the origin followed by a high intermediate barrier. As $\lambda$ decreases towards $0+$, the well becomes deeper, narrower and closer to the origin, but the intermediate barrier height increases. Eq. (11) is solved numerically by the Runga-Kutta algorithm subject to $\psi_{E}^{(1)}=0$. Phase shift is calculated for both the original and isospectral potential (for different values of $\lambda$ ) using Eq. (12). Calculated phase shifts in the range $0<E<1$ have been presented in table 1. From the results, it is clear that the phase shift produced by the original potential is same as that produced by the isospectral potential for the same $l$ value within numerical errors. For smaller $\lambda$, the isospectral potential $\hat{V}_{\text {eff }}(r ; \lambda)$ has a very sharp well near the origin and a very high intermediate barrier which causes a numerical error in evaluating the wave function $\psi_{E}^{(1)}$ by solving Eq. (11). Moreover, as the original potential and the isospectral potential produce same phase shift corresponding to a particular energy and $l$ value, the phase shift relation [Eq. (2)] is not valid for isospectral potentials whereas it is valid for supersymmetric partner potentials $[6,7]$.

\section{CONCLUSIONS}

In this work, the relationship between phase shifts produced by two isospectral potentials has been critically examined. Although the energy eigenvalues, reflection and transmission coefficients are same for isospectral poten- 
tials, the phase shift relation between them was not investigated earlier. In this work, I have tried to find an answer to this question. From the numerical results, it has been established that the isospectral potentials produce same phase shift corresponding to same ' $l$ ' value at a given energy. The well known phase shift relation (Eq. (2)), which is valid for all supersymmetric partner potentials (for both SIP and non-SIP) [6,7], is not valid for isospectral potentials. An example is provided here for a finite square well potential which is non-SIP.

Acknowledgement. The author acknowledges the support by way of financial assistance from the University Grants Commission (UGC), India in terms of a Minor Research project, vide sanction no. F. PSW 074/13-14 (ERO).
[1] E. Witten, Nucl. Phys. B 188, 513 (1981).

[2] F. Cooper, B. Freedman, Ann. Phys. 146, 262 (1983).

[3] Y. A. Gol'fand, E. P. Likhtman, J. Exp. Theor. Phys. Lett. 13, 323 (1971).

[4] F. Cooper, A. Khare, U. Sukhatme, Phys. Rep. 251, 267 (1995).

[5] C. V. Sukumar, J. Phys. A 18, 2937 (1985).
[6] S. Mahapatra, T. K. Das, Mod. Phys. Lett. A 26, 1753 (2011).

[7] S. Mahapatra, J. Mod. Phys. 3, 74 (2012).

[8] M. M. Nieto, Phys. Lett. B 145, 208 (1984).

[9] M. Abramowitz, I. A. Stegun, Handbook of Mathematical Functions (Dover Publication, 1965).

\title{
ФАЗОВИЙ ЗСУВ ІЗОСПЕКТРАЛЬНИХ ПОТЕНЦІАЛІВ
}

\author{
Саб'ясачі Магапатра \\ Коледж катедральної місіӥ ім. Св. Павла, Калькутта, Індія
}

Критично проаналізовано фазовий зсув, який дають ізоспектральні потенціали. Вони можуть значно відрізнятися формою від оригінальних потенціалів, маючи водночас ідентичні спектри. У цій праці показано, що ізоспектральні потенціали за заданої енергї̈ дають той самий фазовий зсув, що й оригінальні. 